Published in final edited form as:

Hum Gene Ther. 2007 January ; 18(1): 81-87.

\title{
Hybrid Adeno-Associated Virus Bearing Nonhomologous Inverted Terminal Repeats Enhances Dual-Vector Reconstruction of Minigenes In Vivo
}

\author{
ZIYING YAN $^{1,2}$, DIANA C.M. LEI-BUTTERS ${ }^{1}$, YULONG ZHANG ${ }^{1}$, ROMAN ZAK ${ }^{1}$, and JOHN F. \\ ENGELHARDT $1,2,3$ \\ 1 Department of Anatomy and Cell Biology, University of Iowa School of Medicine, Iowa City, IA 52242 \\ 2 Center for Gene Therapy, University of Iowa School of Medicine, Iowa City, IA 52242 \\ 3 Department of Internal Medicine, University of Iowa School of Medicine, Iowa City, IA 52242
}

\begin{abstract}
We have previously demonstrated that hybrid adeno-associated viral (AAV) vectors bearing nonhomologous inverted terminal repeats (ITRs) enhance directional intermolecular recombination and the efficiency of dual-AAV vector trans-splicing in cultured cells. Using hybrid-ITR vectors carrying two exons of a lac $Z$ minigene, we demonstrate that this dual-vector approach also mediates higher levels (3- to 6-fold) of gene reconstitution in mouse skeletal muscle, liver, and heart. Inhibition of the proteasome by systemic administration of Doxil (Food and Drug Administration-approved lipid-formulated doxorubicin) further enhanced dual-vector trans-splicing 6- to 12-fold in two mouse strains. Hence, using hybrid-ITR AAV vectors in combination with proteasome modulation enhanced dual-vector delivery of a transgene $\sim 36$-fold over the current dual-vector trans-splicing approaches. These data provide in vivo evidence that ITR sequence-dependent homologous recombination, rather than nonhomologous end joining, is the predominant mechanism for AAV genome heterodimerization. Hence, enhanced directional recombination provided by hybrid-ITR vectors may be a useful in vivo strategy for improving dual-vector delivery of transgenes larger than the AAV packaging limit.
\end{abstract}

\section{INTRODUCTION}

Adeno-associated virus (AAV) is an extremely promising vector for in vivo delivery of transgene cassettes up to $5 \mathrm{~kb}$ in length (Berns and Giraud, 1996; Carter, 2000). This package capacity has been effectively doubled by the development of a dual-vector trans-splicing approach (Yan et al., 2002; Duan et al., 2003b). In this approach, an oversized transgene expression cassette is engineered into a two-exon minigene and each exon is codelivered to the same cell by two independent AAV vectors. After intermolecular recombination between two independent AAV genomes, reconstitution of the minigene cassette allows for intermolecular trans-splicing of the gene product. Such a dual-AAV approach has been successfully tested with reporter genes in mouse muscle (Duan et al., 2000a; Sun et al., 2000; Yan et al., 2000), liver (Nakai et al., 2000), and eyes (Reich et al., 2003). These studies have paved the way for the use of dual-vector trans-splicing in human gene therapy. Indeed, the effectiveness of this dual-AAV vector strategy has been demonstrated for the large (6-kb) minidystrophin gene-with the achievement of therapeutic levels of minidystrophin

Address reprint requests to: Dr. John F. Engelhardt, Department of Anatomy and Cell Biology, Room 1-111 BSB, University of Iowa, 51 Newton Road, Iowa City, IA 52242-1109, E-mail: john-engelhardt@uiowa.edu. 
expression in the $m d x$ mouse model of Duchenne muscular dystrophy (Lai et al., 2005; Liu et al., 2005).

Despite the promising prospects of dual-rAAV vector approaches, the level of gene expression obtained with the current system remains substantially lower than for delivery of a transgene encoded within a single vector. Two factors that limit the efficiency of this approach are the heterodimerization rate of the two input vector genomes and the directionality of the recombination event. For example, when both exons of the minigene cassette are in the same orientation within the two vector genomes, only those heterodimers formed in a head-to-tail manner are active substrates for trans-splicing minigene mRNA products (Yan et al., 2000).

The development of hybrid rAAV vectors with nonhomologous type 2 and type 5 inverted terminal repeats (ITRs) has enhanced our ability to control genome circularization and concatemerization in a manner that enhances directional intermolecular recombination compatible with the dual-vector trans-splicing of transgenes (Yan et al., 2005). In comparison with homologous AAV2 or AAV5 ITR vectors, hybrid 2:5-ITR vectors have been shown to less efficiently form circular monomers through self-circularization, and to more effectively undergo directional intermolecular recombination. With a preferred recombinational bias to form head-to-tail heterodimers, the hybrid 2:5-ITR vectors were found to be 6- to 10-fold more efficient at reconstituting a lac $Z$ minigene than homologous 2:2-or 5:5-ITR vectors after coinfection in HeLa and primary fetal fibroblasts (Yan et al., 2005). These encouraging in vitro results prompted us to assess whether this hybrid-ITR dual-vector trans-splicing approach could also enhance in vivo gene delivery to several organs in mouse models.

\section{MATERIALS AND METHODS}

\section{AAV vectors}

Previously described homologous-ITR and hybrid-ITR AAV vectors harboring either the enhanced green fluorescent protein (eGFP) or two exons of a lacZ minigene were used in these studies (Yan et al., 2005). These vector genomes contained either homologous 2:2-ITR and 5:5-ITR sequences derived from AAV2 and AAV5 serotypes, respectively, or hybrid 2:5-ITR sequences from both serotype ITRs. All viruses were pseudotyped in AAV type 1 capsids and purified through iodixanol ultracentrifuge fractionation, followed by high-performance liquid chromatography (HPLC) on a POROS PI anion-exchange column (Applied Biosystems, Foster City, CA) as previously described (Yan et al., 2006). Using previously described nomenclature (Yan et al., 2005), AAV type 1 viruses used in these studies included the following: AV2:2/1eGFP, AV5:5/1eGFP, AV2:5/1eGFP, AV2:2/1LacZ-donor, AV2:2/1LacZacceptor, AV5:2/1LacZdonor, and AV2:5/1LacZacceptor.

\section{In vivo infection procedures in mice}

AV2:2/1eGFP, AV5:5/1eGFP, and AV2:5/1eGFP vectors were evaluated after direct injection of $10^{10}$ particles into the anterior tibialis muscle of mice or, alternatively, after infection of the liver by tail vein injection with $2 \times 10^{11}$ particles. GFP transgene expression was assessed 2 weeks postinfection. The two sets of lacZ trans-splicing viruses as shown in Fig. 2A, including homologous-ITR vectors (AV2:2LacZdonor and AV2:2LacZacceptor) and the hybrid-ITR vectors (AV5:2LacZ-donor and AV2:5LacZacceptor), were compared for their ability to reconstitute lac $Z$ minigene expression in tibialis muscle, liver, heart, and lung. In these studies the donor and acceptor vectors were delivered either individually or in combination by either local injection $\left(5 \times 10^{10}\right.$ particles of each) or systemic delivery by tail vein injection $\left(10^{12}\right.$ particles of each) as previously described (Yan et al., 2000,2004). C57BL/6J mice and

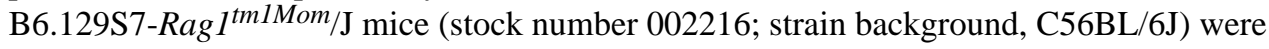


purchased from Jackson Laboratory (Bar Harbor, ME). All animal experiments were performed in accordance with National Institutes of Health (NIH, Bethesda, MD) regulatory guidelines.

\section{$\beta$-Galactosidase assays}

In all studies, transgene expression was assessed 2 weeks postinfection and $\beta$-galactosidase expression was assessed using two independent assays. Isolated tissue and organs were either lysed in reporter lysis buffer (Promega, Madison, WI) to generate tissue extracts, or fresh frozen for sectioning. $\beta$-Galactosidase expression was evaluated by in situ 5-bromo-4-chloro-3indolyl- $\beta$-D-galactopyranoside (X-Gal) staining and in an enzymatic assay using the GalactoLight Plus system (Applied Biosystems), as previously described (Duan et al., 2001). For the enzymatic assay, tissue extract supernatant was heated at $48^{\circ} \mathrm{C}$ for $1 \mathrm{hr}$ to inactivate endogenous $\beta$-galactosidase activity. Morphometric quantification of in situ X-Gal staining of tissue sections was performed, with NIH ImageJ software, on 15 randomly captured photomicrographs for each experimental point. This was used to calculate the mean relative percent LacZ-positive area for each vector combination.

\section{RESULTS AND DISCUSSION}

It is currently unclear whether AAV ITR primary sequence and/or secondary structure influences heterodimerization of AAV genomes in vivo. In vitro evidence supports the importance of ITR primary sequence in the intermolecular recombination of viral genomes (Yan et al., 2005), whereas in vivo evidence from liver suggests that nonhomologous end joining is the primary mechanism for vector genome concatemerization (Chen et al., 2001; Nakai et al., 2003; Grimm et al., 2006). The present study sought to evaluate whether ITR primary sequence influences intermolecular recombination in vivo, using a trans-splicing dual vector genetic approach.

Evidence suggests that AAV-mediated transduction of the liver is influenced primarily by capsid serotype rather than the genotype of the ITR (Grimm et al., 2006). Because our approach to investigate intermolecular recombination in vivo uses both AAV5 and AAV2 ITRs, and these two vector genotypes have yet to be directly compared within an AAV1 capsid serotype, we first needed to confirm that AAV2 and/or AAV5 ITR-containing genomes mediate similar levels of gene expression from a single transgene cassette in vivo. To this end, mouse tibialis muscle and liver were infected with virus containing 2:2-, 5:5-, or 2:5-ITR viral genomes and an eGFP reporter transgene cassette. All viruses were pseudotyped in AAV type 1 capsids, and eGFP expression was assessed 2 weeks postinfection. All three viruses led to the production of similar levels of eGFP expression in mouse muscle (Fig. 1) and liver (data not shown). These findings demonstrate that AAV2 and AAV5 ITRs facilitate similar levels of rAAV transduction and genome conversion to expression-competent double-stranded forms in vivo. This is consistent with a report demonstrating that AAV transduction is restricted primarily by capsid serotype rather than ITR genotype, in liver (Grimm et al., 2006). Previous studies in HeLa cells have also reported that levels of intermolecular recombination (as determined by the level of a trans-spliced lacZ minigene product) are the same when either 2:2-ITR or 5:5-ITR homologous-ITR vector sets are used (Yan et al., 2005), demonstrating that both the AAV2 and AAV5 ITR genotypes undergo homologous recombination to similar extents. On the basis of these findings, we focused on comparing the heterodimerization efficiencies of only the homologous 2:2-ITR and hybrid 2:5-ITR vector sets. Our approach used previously described (Yan et al., 2005) homologous-ITR (AV2:2LacZdonor and AV2:2LacZacceptor) and hybridITR (AV5:2LacZdonor and AV2:5LacZacceptor) vector genomes (Fig. 2A), which had been pseudotyped in AAV1 capsids, to evaluate heterodimerization efficiencies in vivo, using a previously described genetic approach of reconstituting a trans-spliced lac $Z$ minigene product (Duan et al., 2001). 
Heterodimerization of AAV2 genomes has been studied extensively in mouse skeletal muscle (Yang et al., 1999; Sun et al., 2000; Yan et al., 2000; Duan et al., 2003a). This tissue has also been used most frequently to study dual-vector trans-splicing approaches (Yan et al., 2000; Lai et al., 2005; Liu et al., 2005), and as such was the first chosen for in vivo analysis of hybridITR vectors. For both the hybrid-ITR (AV5:2/1LacZ-donor and AV2:5/1LacZacceptor) and homologous-ITR (AV2:2/1LacZdonor and AV2:2/1LacZacceptor) vector sets, equal amounts of donor and acceptor virus were premixed and used for the coinfection of mouse anterior tibialis by local injection. Age-matched control mice were infected with the individual donor or acceptor virus only. Two weeks postinfection, muscles were harvested to examine $\beta$ galactosidase expression. Results from this comparison demonstrated a 4-fold increase in trans-spliced lacZ gene product from the hybrid-ITR vector set as compared with homologousITR vectors (Fig. 2B). No $\beta$-galactosidase activity was detected for any of the four transsplicing vectors that were delivered individually to muscle (Fig. 2B). These results substantiate in vitro findings, in HeLa cells, demonstrating the improved performance of hybrid 2:5-ITR vectors in reconstituting a lac $Z$ minigene product by virtue of enhanced directional heterodimerization (Yan et al., 2005).

We have previously demonstrated that coadministration of a proteasome inhibitor ( $\mathrm{N}$-acetyl1-leucyl-1-leucyl-1-norleucine [LLnL] or doxorubicin) can significantly enhance AAV transduction in vitro and in vivo by promoting intracellular trafficking of virus to the nucleus (Duan et al., 2000b; Ding et al., 2003; Yan et al., 2004). We have also observed that proteasome inhibitors, such as doxorubicin, can enhance the efficiency of AAV2 trans-splicing vectors in a number of cell lines (HeLa, 293, and primary fetal fibroblasts; our unpublished data). Thus, we sought to assess whether the efficiency of AAV trans-splicing in vivo is enhanced by modulating the proteasome pathway. Because Doxil (U.S. Food and Drug Administrationapproved lipid-formulated doxorubicin) has been shown to enhance in vivo gene transfer with AAV (Yan et al., 2004), we administered this drug systemically by tail vein administration, and evaluated the effects of this treatment on dual-vector trans-splicing with rAAV in muscle. In these studies, $200 \mu \mathrm{g}$ of Doxil was administered at the time of C57BL/6 mouse muscle infection with either hybrid- or homologous-ITR vector sets. Doxil significantly enhanced LacZ expression ( $\sim 9$ fold) for both vector sets (Fig. 2 C), whereas the 4-fold greater efficiency of hybrid-ITR vector trans-splicing, as compared with homologous-ITR trans-splicing vectors, was unaffected by Doxil. Similar findings of enhanced hybrid vector trans-splicing in tibialis muscle were also observed in C57BL/6 Ragl-deficient mice in the presence of Doxil (Fig. 3A); enzymatic assays revealed 6-fold higher $\beta$-galactosidase activity in muscle extracts from the hybrid vector infections as compared with homologous-ITR vectors. These studies on the Ragl knockout (KO) background also assessed whether the enhanced reconstitution of lac $Z$ gene product, using hybrid vectors, was the result of increased numbers of transgeneexpressing cells or enhanced product expression in a similar numbers of cells. X-Gal staining of muscle tissue sections demonstrated that LacZ expression from the hybrid vector set enhanced both the number of positive myofibers and the intensity of transgene expression (Fig. 3B). The comparison of hybrid- and homologous-ITR trans-splicing vectors in Ragl-KO mice is also useful because it obviates potential acquired immunity to the lac $Z$ transgene, which might be affected by the level of lac $Z$ gene expression. Overall, this demonstration of enhanced directional recombination of AAV genomes in muscle by hybrid-ITR vectors suggests that homologous recombination of viral genomes in this tissue is directly influenced by the sequence of the ITR.

Previous studies have suggested that the molecular fate of AAV genomes in the liver is somewhat different from that in muscle, implicating ITR sequence-independent nonhomologous end joining of AAV genomes as a mechanism for intermolecular concatemerization in hepatocytes (Chen et al., 2001; Nakai et al., 2003; Grimm et al., 2006). Because dual-AAV vector trans-splicing has also been reported in mouse liver (Nakai et al., 
2000), it is currently unclear whether independent mechanisms for AAV genome concatemerization control dual-vector trans-splicing in liver and muscle. We hypothesized that AAV heterodimerization in the liver is controlled by nonhomologous end joining of viral genomes, and that there should therefore be no difference in trans-splicing efficiencies between hybrid-and homologous-ITR AAV vector sets. To test this hypothesis, C57BL/6 Ragl-KO mice were infected with $10^{12}$ particles of either the hybrid-ITR or homologous-ITR vector sets, by tail vein injection together with $200 \mu \mathrm{g}$ of Doxil. In these experiments, hybrid-ITR vectors produced 4-fold greater levels of $\beta$-galactosidase activity in tissue extracts and 7 -fold greater levels of X-Gal-positive histochemical staining in tissue sections, as compared with homologous-ITR vectors (Fig. 4). These data suggest that, in contrast to our original hypothesis, ITR-mediated homologous recombination in the liver is sequence dependent and critical for intermolecular concatemerization of AAV genomes. The findings suggest that the mechanisms of genome concatemerization in liver and muscle are similar, and that directional recombination between homologous ITRs within hybrid-ITR donor and acceptor vectors can be used to enhance trans-splicing efficiency in both tissues.

Systemic delivery of trans-splicing AAV vectors to the liver by tail vein injection also provided the opportunity to assess intermolecular concatemerization in other organs. We assessed $\beta$ galactosidase activity in tissue extracts from both heart and lung from the same mice that were evaluated for liver transgene expression. Results from these experiments demonstrated a 5.3fold greater level of $\beta$-galactosidase activity in heart from the hybrid-ITR vector set, as compared with the activity generated by the homologous-ITR vectors (Fig. 5). However, dualvector reconstitution of the lac $Z$ minigene was undetectable in lung extracts from animals infected with either vector set and resulted in levels of $\beta$-galactosidase activity similar to those seen in control animals that had received only the donor or acceptor vector (data not shown). Interested by the lack of detectable lac $Z$ minigene expression mediated by genome heterodimerization in lung, we hypothesized that the processing and control of viral genome concatemerization in the lung might be different than in muscle and liver. Thus, we also performed experiments in which C57BL/6 Ragl-KO mice were infected by intranasal instillation with both sets of trans-splicing vectors at a multiplicity of infection (MOI) of $10^{11}$ particles/mouse in the presence of Doxil as described previously (Yan et al., 2004). Despite the fact that the AAV1 serotype is known to effectively transduce mouse lung (Yan et al., 2006) and Doxil also enhances AAV transduction in the lung (data not shown), no detectable trans-spliced lacZ gene product was observed in lung lysates. These findings suggest that cell type-specific differences in AAV genome conversion in the lung may limit the application of dual-AAV vector trans-splicing approaches for this organ.

Although lacZ minigene reconstitution with two independent input vectors is an indirect indicator of viral genome heterodimerization, we also attempted to confirm the extent of genome heterodimerization by performing Southern blots of Hirt DNA isolated from muscle, liver, and lung, using a fraction of the organ evaluated in the expression studies. However, we were unable to visualize molecular forms consistent with heterodimerization; predominant signal came from linear input viral DNA (data not shown). We interpret this finding to reflect the fact that a minority of genomes are transcriptionally competent in all organs tested and below the sensitivity of the Southern blot assay. Nonetheless, using the genetic approach (i.e., lac $Z$ minigene reconstitution), the lung was by far the least effective at supporting heterodimerization, despite the ability of AAV1 to effectively transduce all the tissues tested using a single transgene cassette.

In summary, the in vivo study described here, together with our earlier in vitro data, clearly demonstrate a critical role for ITR sequences in AAV genome heterodimerization. The use of hybrid-ITR vectors can significantly enhance the efficacy of dual-AAV vector trans-splicing 
approaches in skeletal muscle, liver, and cardiac muscle. Such methods may aid in the application of this approach to deliver transgenes larger than the AAV packaging capacity.

\section{Acknowledgements}

This work was supported by the NIH (grant RO1 HL58340; J.F.E.), the Center for Gene Therapy (DK54759), and Targeted Genetics Corporation.

\section{References}

BERNS, KI.; GIRAUD, C. Adeno-associated Virus (AAV) Vectors in Gene Therapy. Springer-Verlag; Berlin: 1996.

CARTER BJ. Adeno-associated viruses as gene transfer vehicles. Contrib Microbiol 2000;4:85-106. [PubMed: 10941572]

CHEN ZY, YANT SR, HE CY, MEUSE L, SHEN S, KAY MA. Linear DNAs concatemerize in vivo and result in sustained transgene expression in mouse liver. Mol Ther 2001;3:403-410. [PubMed: 11273783]

DING W, YAN Z, ZAK R, SAAVEDRA M, RODMAN DM, ENGELHARDT JF. Second-strand genome conversion of adeno-associated virus type 2 (AAV-2) and AAV-5 is not rate limiting following apical infection of polarized human airway epithelia. J Virol 2003;77:7361-7366. [PubMed: 12805434]

DUAN D, YUE Y, YAN Z, ENGELHARDT JF. A new dual-vector approach to enhance recombinant adeno-associated virus-mediated gene expression through intermolecular cis activation. Nat Med 2000a;6:595-598. [PubMed: 10802719]

DUAN D, YUE Y, YAN Z, YANG J, ENGELHARDT JF. Endosomal processing limits gene transfer to polarized |airway epithelia by adeno-associated virus. J Clin Invest 2000b;105:1573-1587. [PubMed: 10841516]

DUAN D, YUE Y, ENGELHARDT JF. Expanding AAV packaging capacity with trans-splicing or overlapping vectors: A quantitative comparison. Mol Ther 2001;4:383-391. [PubMed: 11592843]

DUAN D, YUE Y, ENGELHARDT JF. Consequences of DNA-dependent protein kinase catalytic subunit deficiency on recombinant adeno-associated virus genome circularization and heterodimerization in muscle tissue. J Virol 2003a;77:4751-4759. [PubMed: 12663782]

DUAN D, YUE Y, ENGELHARDT JF. Dual vector expansion of the recombinant AAV packaging capacity. Methods Mol Biol 2003b;219:29-51. [PubMed: 12596997]

GRIMM D, PANDEY K, NAKAI H, STORM TA, KAY MA. Liver transduction with recombinant adeno-associated virus is primarily restricted by capsid serotype not vector genotype. J Virol 2006;80:426-439. [PubMed: 16352567]

LAI Y, YUE Y, LIU M, GHOSH A, ENGELHARDT JF, CHAMBERLAIN JS, DUAN D. Efficient in vivo gene expression by trans-splicing adeno-associated viral vectors. Nat Biotechnol 2005;23:14351439. [PubMed: 16244658]

LIU M, YUE Y, HARPER SQ, GRANGE RW, CHAMBERLAIN JS, DUAN D. Adeno-associated virusmediated microdystrophin expression protects young $m d x$ muscle from contraction-induced injury. Mol Ther 2005;11:245-256. [PubMed: 15668136]

NAKAI H, STORM TA, KAY MA. Increasing the size of rAAV-mediated expression cassettes in vivo by intermolecular joining of two complementary vectors [see comments]. Nat Biotechnol 2000;18:527-532. [PubMed: 10802620]

NAKAI H, MONTINI E, FUESS S, STORM TA, MEUSE L, FINEGOLD M, GROMPE M, KAY MA. Helper-independent and AAV-ITR-independent chromosomal integration of double-stranded linear DNA vectors in mice. Mol Ther 2003;7:101-111. [PubMed: 12573623]

REICH SJ, AURICCHIO A, HILDINGER M, GLOVER E, MAGUIRE AM, WILSON JM, BENNETT J. Efficient trans-splicing in the retina expands the utility of adeno-associated virus as a vector for gene therapy. Hum Gene Ther 2003;14:37-44. [PubMed: 12573057]

SUN L, LI J, XIAO X. Overcoming adeno-associated virus vector size limitation through viral DNA heterodimerization. Nat Med 2000;6:599-602. [PubMed: 10802720] 
YAN Z, ZHANG Y, DUAN D, ENGELHARDT JF. From the Cover: Trans-splicing vectors expand the utility of adeno-associated virus for gene therapy. Proc Natl Acad Sci USA 2000;97:6716-6721. [PubMed: 10841568]

YAN Z, RITCHIE TC, DUAN D, ENGELHARDT JF. Recombinant AAV-mediated gene delivery using dual vector heterodimerization. Methods Enzymol 2002;346:334-357. [PubMed: 11883078]

YAN Z, ZAK R, ZHANG Y, DING W, GODWIN S, MUNSON K, PELUSO R, ENGELHARDT JF. Distinct classes of proteasome-modulating agents cooperatively augment recombinant adenoassociated virus type 2 and type 5-mediated transduction from the apical surfaces of human airway epithelia. J Virol 2004;78:2863-2874. [PubMed: 14990705]

YAN Z, ZAK R, ZHANG Y, ENGELHARDT JF. Inverted terminal repeat sequences are important for intermolecular recombination and circularization of adeno-associated virus genomes. J Virol 2005;79:364-379. [PubMed: 15596830]

YAN Z, LEI-BUTTERS DC, LIU X, ZHANG Y, ZHANG L, LUO M, ZAK R, ENGELHARDT JF. Unique biologic properties of recombinant AAV1 transduction in polarized human airway epithelia. J Biol Chem 2006;281:29684-29692. [PubMed: 16899463]

YANG J, ZHOU W, ZHANG Y, ZIDON T, RITCHIE T, ENGELHARDT JF. Concatamerization of adeno-associated viral circular genomes occurs through intermolecular recombination. J Virol 1999;73:9468-9477. [PubMed: 10516055] 

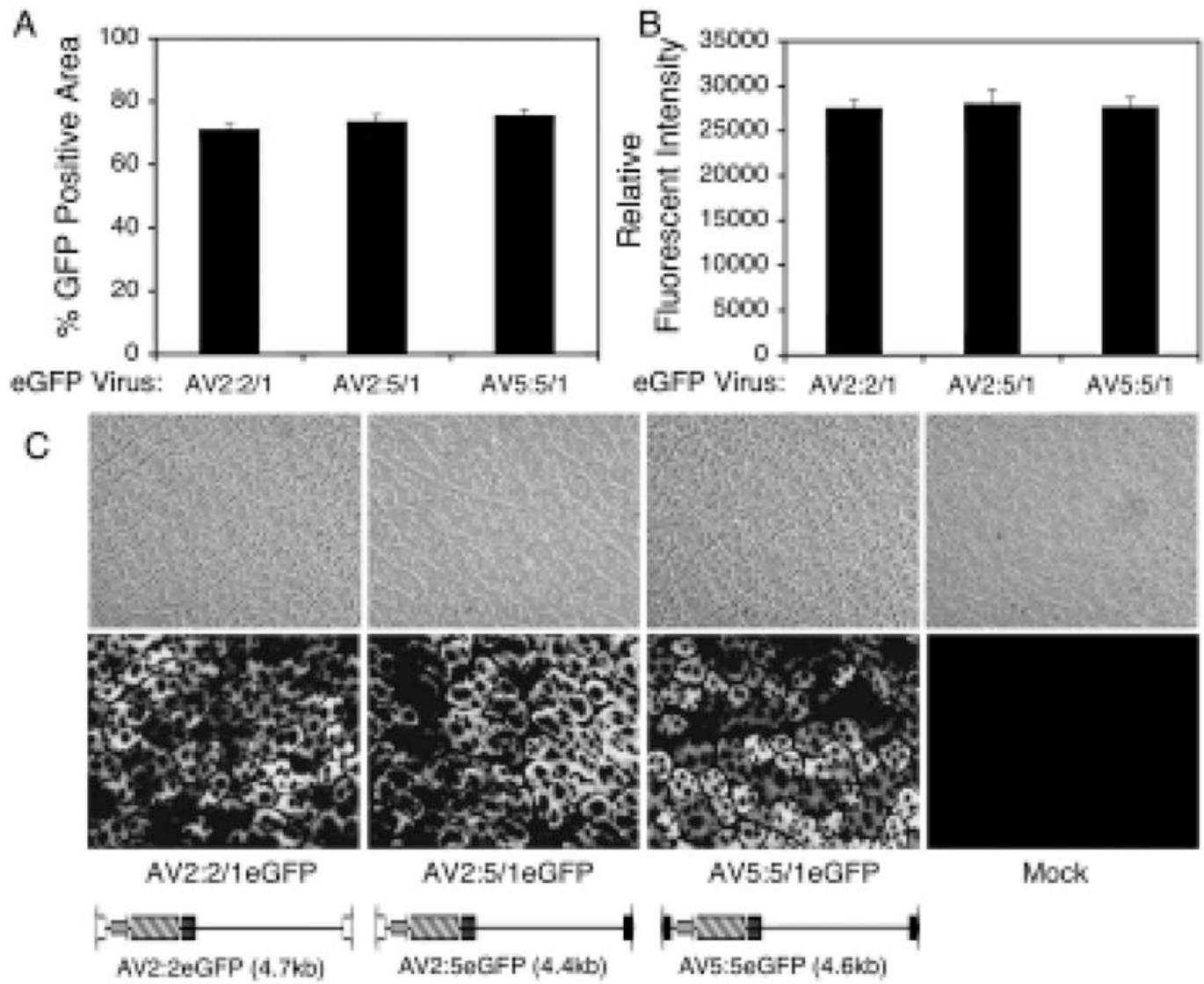

AV5:5/1eGFP
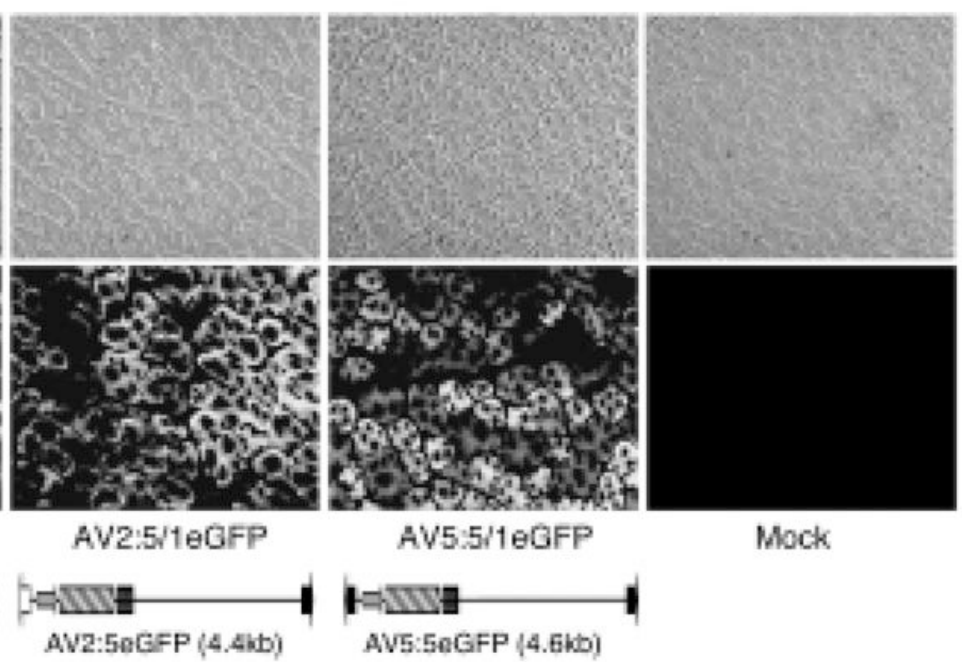

Mock

FIG. 1.

Efficiency of rAAV transduction in mouse muscle is not affected by AAV viral ITR genotype. AV2:2/1eGFP, AV5:5/1eGFP, or AV2:5/1eGFP ( $10^{10}$ particles) was used to infect mouse anterior tibialis by direct local injection. GFP expression in mouse muscle sections was quantified 2 weeks postinfection as (A) the percentage eGFP-positive area and (B) the relative fluorescence intensity (positive GFP area multiplied by the mean fluorescence intensity) with NIH ImageJ software. (C) Representative photomicrographs of muscle sections for each of the depicted vectors. 
A

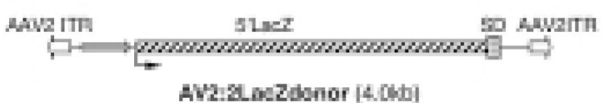

Ahreith sa sume? brimowese

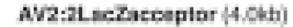

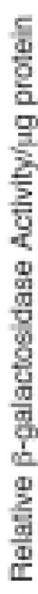

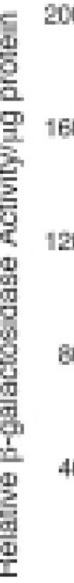

A.MTTF

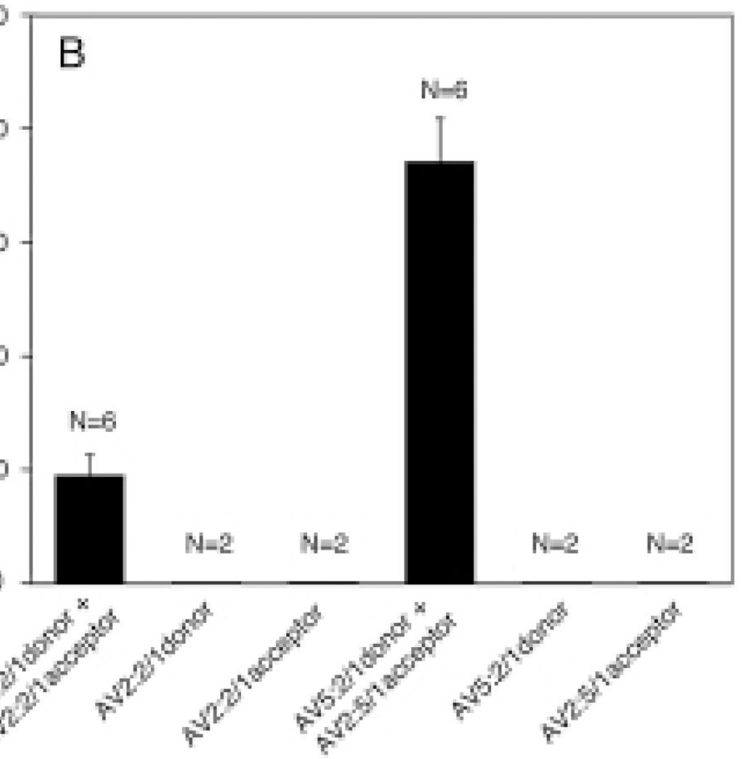

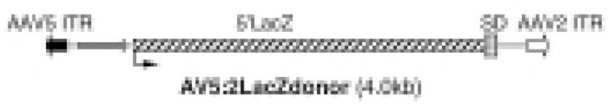

Whatr Bn Jice 苚

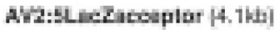
M⿻上5 ITF

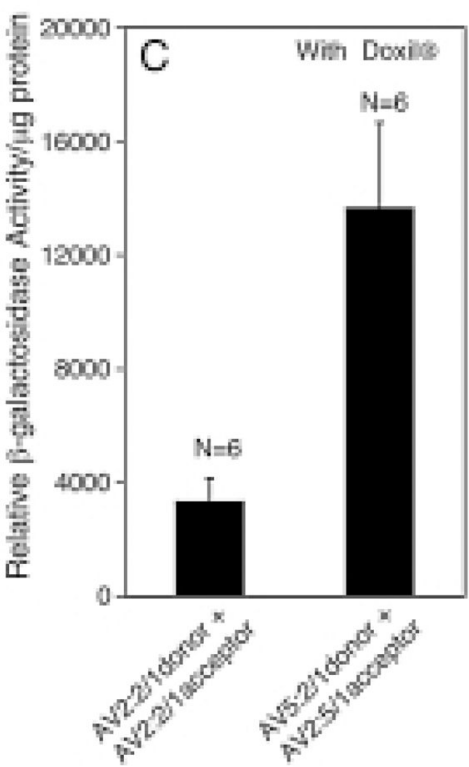

FIG. 2.

Efficiency of vector genome heterodimerization with homologous- and hybrid-ITR AAV1 viruses in muscle. (A) Schematic representation of the homologous- and hybrid-ITR lacZ trans-splicing vector viral genomes used in this study. (B and $\mathbf{C})$ Comparison of dual-vector heterodimerization in C57BL/6 mouse anterior tibialis muscle after infection with homologous- and hybrid-ITR vector genomes pseudotyped in AAV1 capsid. Infections were performed in the absence $(\mathbf{B})$ and in the presence $(\mathbf{C})$ of proteasome inhibitor Doxil. Enzymatic quantification of $\beta$-galactosidase activity in tissue lysates was performed 2 weeks postinfection. Data represent mean (+SEM) $\beta$-galactosidase activity of six independent infections for each group. 

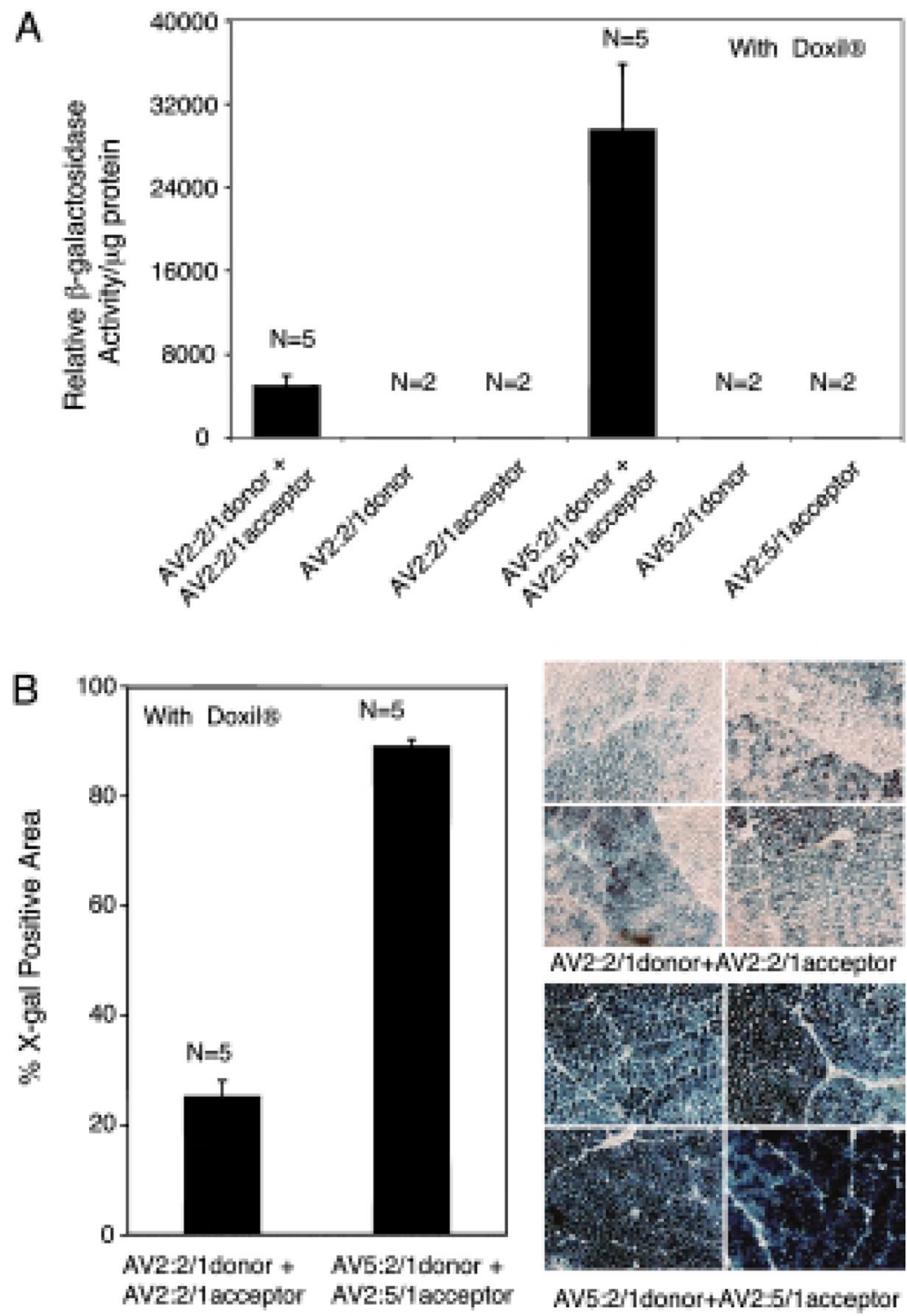

FIG. 3.

Hybrid-ITR vector genomes enhance directional heterodimerization after infection of C57BL/ 6 Ragl-KO mouse anterior tibialis muscle. Directional genome heterodimerization was assessed after tibialis infection with the indicated viral combinations in the presence of Doxil. (A) $\beta$-Galactosidase enzymatic assays performed on tissue lysates 2 weeks postinfection. Data represent mean (+SEM) $\beta$-galactosidase activity of two to five independent infections for each group as indicated. (B) Morphometric quantification of X-Gal-stained mouse muscle from the indicated infections (left) and representative photomicrographs of the X-Gal-stained muscle sections $(r i g h t)$. Data represents mean (+SEM) percent X-Gal-positive area observed in liver sections ( $n=5$ mice for each experimental group). 
A
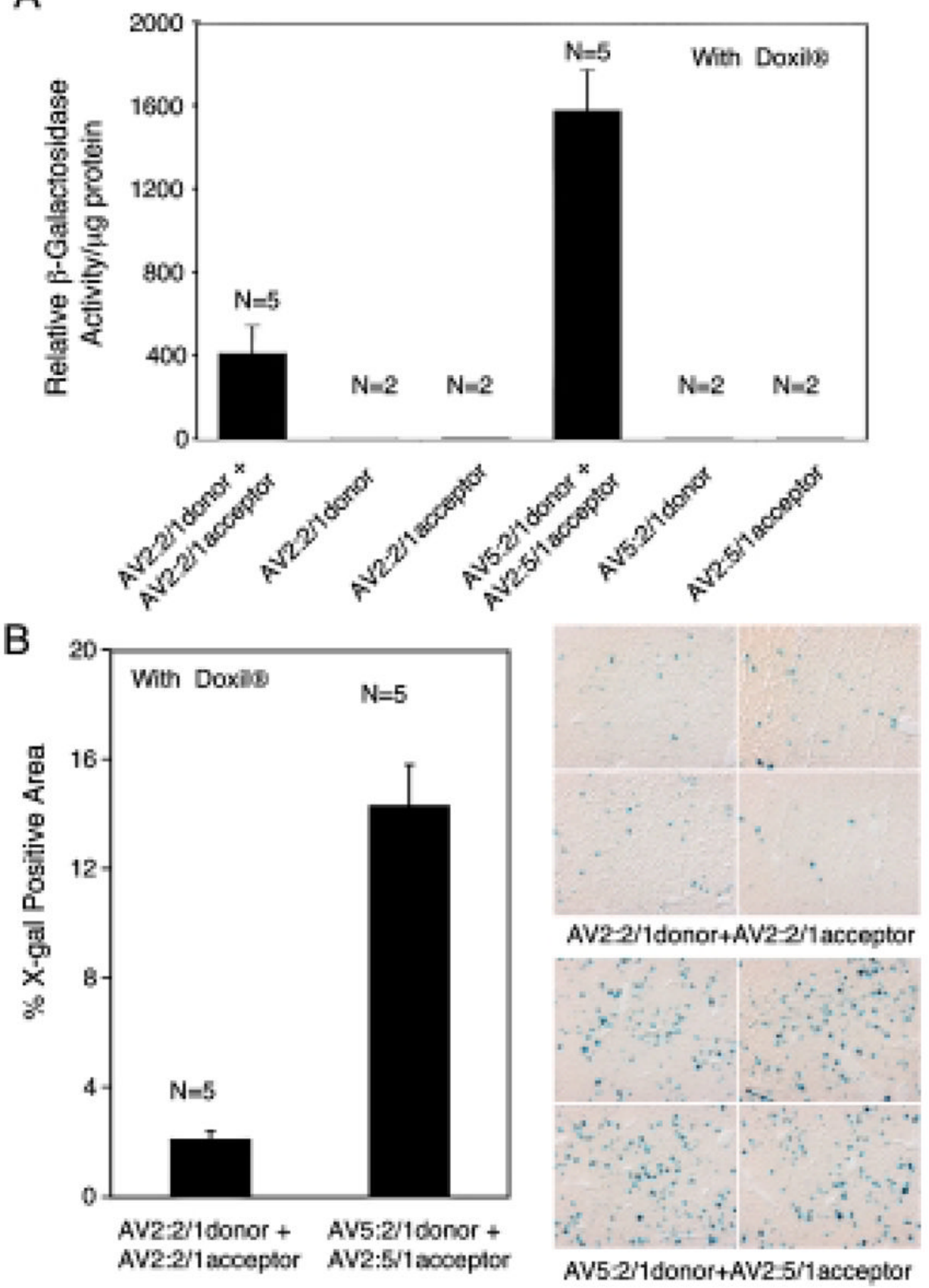

FIG. 4.

Hybrid-ITR vector genomes enhance directional heterodimerization after infection of C57BL/ 6 Rag1-KO mouse liver. Directional genome heterodimerization was assessed in the liver after tail vein injection of the indicated viral combinations in the presence of Doxil. (A) $\beta$ -

Galactosidase enzymatic assays performed on tissue lysates 2 weeks postinfection. Data represent mean $( \pm$ SEM $\beta$-galactosidase activity of two to five independent infections for each group as indicated. (B) Morphometric quantification of X-Gal-stained mouse liver from the indicated infections (left) and representative photomicrographs of the X-Gal-stained liver sections $(r i g h t)$. Data represent mean $( \pm \mathrm{SEM})$ percent X-Gal-positive area observed in liver sections ( $n=5$ mice for each experimental group). 


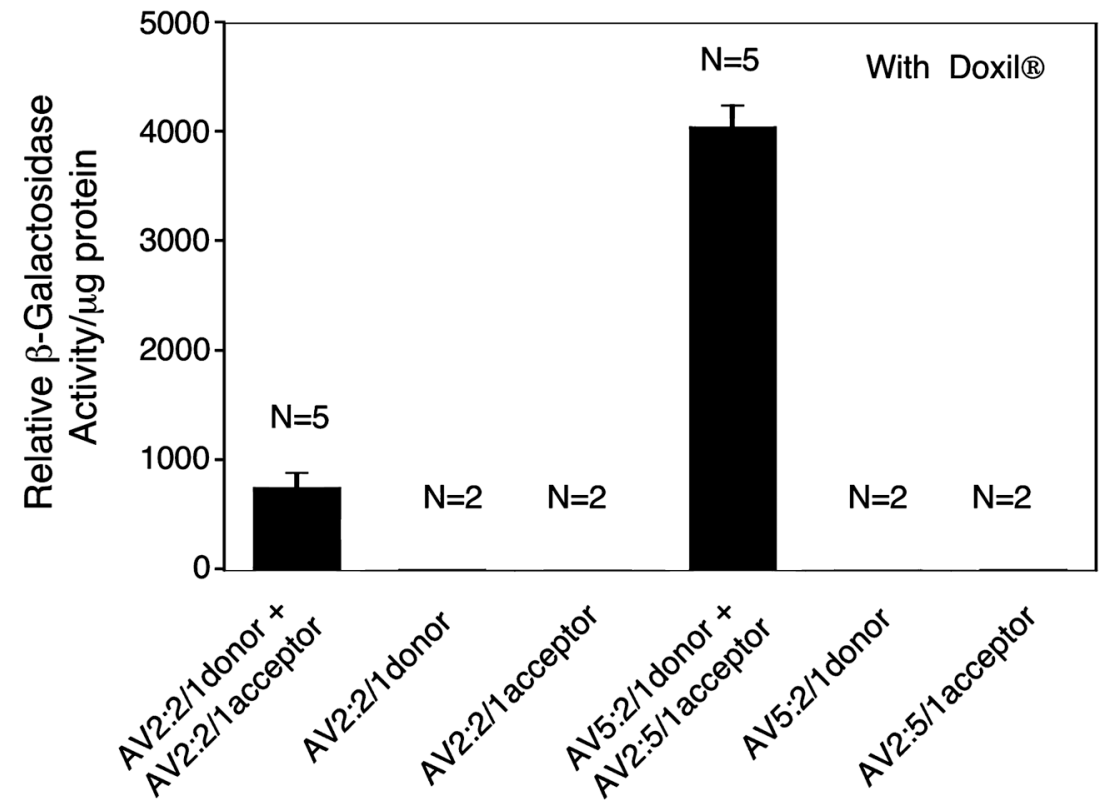

FIG. 5.

Hybrid-ITR vector genomes enhance directional heterodimerization after infection of C57BL/ $6 \mathrm{Rag} 1-\mathrm{KO}$ mouse heart. Directional genome heterodimerization was assessed in the heart after tail vein injection of the indicated viral combinations in the presence of Doxil. $\beta$-Galactosidase enzymatic assays on tissue lysates 2 weeks postinfection are given. Data represent mean $( \pm$ SEM) $\beta$-galactosidase activity of two to five independent infections for each group as indicated. 